\title{
Análisis de accesibilidad y conectividad de la red vial intermunicipal en el microsistema regional de la provincia Centro en Boyacá, Colombia
}

\section{ANALYSIS OF ACCESSIBILITY AND CONNECTIVITY OF THE INTERMUNICIPAL RED ROAD IN THE REGIONAL MICRO-SYSTEM OF THE CENTRO PROVINCE IN BOYACÁ, COLOMBIA}

ANÁLISE DA ACESSIBILIDADE E CONECTIVIDADE DA REDE RODOVIÁRIA INTERMUNICIPAL NO MICROSSISTEMA REGIONAL DA PROVÍNCIA DO CENTRO EM BOYACÁ, COLÔMBIA
Para citar este artículo: Bautista, A. F. (2018). Análisis de accesibilidad y conectividad de la red vial intermunicipal en el microsistema regional de la provincia Centro en Boyacá, Colombia. Perspectiva Geográfica, 23(1), 123-141. doi: $10.19053 / 01233769.8058$
ANDRÉS FELIPE Bautista ${ }^{1}$

$\square$

Recibido:

29 de marzo de 2017

Evaluación:

7 de noviembre de 2017

Aprobación:

3 de marzo de 2018

\section{Resumen}

En este artículo se presentan los resultados del análisis la red vial intermunicipal del microsistema regional de la provincia Centro, en el departamento de Boyacá, efectuado a través de la combinación de diferentes indicadores del análisis de redes, la teoría de grafos y herramientas de los sistemas de información geográfica, como un instrumento útil para el conocimiento de la funcionalidad, la centralidad y los desequilibrios presentes de su configuración espacial. Del análisis se obtuvo una

1 Licenciado en Ciencias Sociales de la Universidad Pedagógica y Tecnológica de Colombia, magíster en Geografía del convenio Universidad Pedagógica y Tecnológica de Colombia e Instituto Geográfico Agustín Codazzi. Correo electrónico: bautistaandresfelipe@gmail.com. 
imagen de la densidad de accesibilidad relativa que permite estimar los escenarios de accesibilidad real e ideal, e identificar patrones espaciales que ponen de presente el grado de desintegración y desigualdades territoriales en términos de las características topológicas de la red vial de transportes.

Palabras clave: análisis topológico, conectividad, indices de accesibilidad, microsistema regional, red vial, provincia Centro.

\section{Abstract}

The purpose of this paper is to analyze the inter-municipal road network of the regional micro-system of the Central Province in the department of Boyacá, throughout the combination of different indicators of network analysis, graph theory and geographic information system tools as useful instruments to the knowledge functionality, centrality and present imbalances on its spatial configuration. From the analysis, an image of the density of relative accessibility is obtained and it allows to estimate the real and ideal accessibility scenarios, identifying spatial patterns that show the degree of disintegration and territorial inequalities in terms of the topological characteristics of the transport road network.

Keywords: Topological analysis, connectivity, accessibility indexes, regional microsystem, road network, province Center.

\section{Resumo}

A rede rodoviária intermunicipal do microssistema regional da província do Centro, no departamento de Boyacá, é analisada, através da combinação de diferentes indicadores de análise de redes, teoria dos grafos e ferramentas dos Sistemas de Informação Geográfica, como uma ferramenta útil para conhecimento da funcionalidade, centralidade e desequilíbrios atuais de sua configuração espacial. A partir da análise, obtém-se uma imagem da densidade de acessibilidade relativa que permite estimar os cenários de acessibilidade real e ideal, identificando padrões espaciais que mostram o grau de desintegração e as desigualdades territoriais em termos das características topológicas da rede viária de transporte.

Palavras-chave: Análise topológica, conectividade, indices de acessibilidade, microssistema regional, rede viária, centro provincial. 


\section{Introducción}

En el sistema de transporte terrestre, la red vial y las infraestructuras aparecen como una temática de interés por sus efectos redistributivos, su contribución a la disminución de los desequilibrios territoriales y los aspectos asociados a su dinámica espacial, que conforman amplios estadios de análisis (Fárinos, 2007; Chías, 2008; Bellet, 2013; Bautista y Cerquera, 2014). Las redes viales y la infraestructura de transporte "tienen una influencia cada vez más relevante tanto para las estructuras y los modelos territoriales, como para las visiones de futuro porque impiden o promueven transformaciones radicales en la organización territorial" (Patiño y Salazar, 2016, p. 81).

Las redes viales son elementos fundamentales en las políticas de desarrollo, toda vez que son expresión y fiel reflejo de la evolución de los territorios. Estos sistemas pueden considerarse potencialmente estratégicos al fungir como verdaderos articuladores del espacio urbano y regional. Su funcionalidad, si bien imbuida en la relación directa con la circulación de personas, bienes y mercancías, se extiende más allá a su carácter polivalente, complementario y asociativo, en la medida en que están diseñadas para atender multiplicidad de propósitos, entre ellos, que se particularice como un valor de desarrollo espacial que estimula la integración territorial (Miralles Guasch, 2013).

La red vial, aunque no es un objeto único y exclusivo de la estructuración del territorio, expresa el grado de relaciones e intercambios que puedan darse en su interior, y abordar su estudio implica no solo discernir la disponibilidad de la red, sino establecer sus relaciones de accesibilidad y conectividad como condiciones indispensables para alcanzar diferentes objetivos y servicios (Martínez-Sánchez, 2012; Escobar y Urazán, 2014). Estas medidas, según Gutiérrez y Gómez (1999), son importantes porque tienen un valor social y económico relacionado con la satisfacción personal e individual y con el mismo desarrollo de las áreas de influencia. $\mathrm{Su}$ análisis aporta al conocimiento de las deficiencias de accesibilidad en relación con centros principales, mayores centralidades y patrones espaciales que permiten identificar desigualdades territoriales en términos de las características de la red de transportes (Loyola y Rivas, 2014).

En general, el análisis de redes e infraestructuras de transporte suele requerir modelos que tienden a sustentarse en la teoría clásica de grafos y el análisis topológico, que vinculan particularmente indicadores de accesibilidad. Un ejemplo de ello puede encontrarse en los trabajos pioneros de Herce (1983) y Dupuy (1998) y en otras experiencias adelantadas por Serrano (2004), Cardozo, Gómez y Parras (2009), Principi (2012), Jingyi y Yifang (2013), Loyola y Rivas (2014) y Cats (2017). No obstante, con el desarrollo de los sistemas de información geográfica (SIG) se facilitaron estos procedimientos a través de la asignación de lenguajes cuantitativos cuyas aplicaciones y herramientas de análisis ampliaron sustancialmente estos estudios (Alvarez-Palau y Aguilar, 2015).

Actualmente, el análisis topológico vincula desarrollos que confinan campos de investigación relacionados con la teoría de la red compleja, centrados principalmente en las propiedades estadísticas de la red (estructura y topología), los modelos de redes y el análisis de las dinámicas y comportamientos emergentes (Jingyi y Yifang, 2013; Lotero y Hurtado, 2014). Si bien estos modelos constituyen campos de medición y predicción soportados particular- 
mente en el análisis de la red de transporte urbano, la vulnerabilidad, la resiliencia de la red vial y la optimización de rutas, el presente ejercicio se fundamenta en el desconocimiento de la dinámica de la red vial intermunicipal en el microsistema regional de la provincia Centro, en el departamento de Boyacá, Colombia, en términos topológicos, y en la estimación de patrones de accesibilidad regional que contribuyan a la comprensión de la funcionalidad del territorio y las potencialidades que resultan de interés para la orientación de políticas de ordenación territorial.

La integración de indicadores de accesibilidad topológica $^{2}$ y la aplicación de herramientas de SIG aparecen como una de las ventanas metodológicas con mayor desarrollo. Sus antecedentes pueden observarse en De Cos Guerra (2004), Cardozo et al. (2009), Xie y Levinson (2009) y Freiria, Ribeiro y Tavares (2015). Se considera la relevancia de estos análisis teniendo presente el proceso complejo y dinámico que representa el sistema de transporte y sus distintas dimensiones, entre ellas, la topológica (Xie y Levinson, 2007). En ese sentido, la combinación de los indicadores de accesibilidad y los estimadores de densidades focales Kernel $^{3}$ configura una de las herramientas más útiles a estos propósitos. El interés por su aplicación radica en que estos métodos permiten enriquecer el análisis de accesibilidad y conectividad a partir de la definición de patrones espaciales que dan cuenta de

2 Según Rodrigue, Comtois y Slack (2009), es el conjunto de medidas empleadas para realizar análisis espacial de la red de transporte. De este conjunto, se pueden nombrar los índices de conectividad, accesibilidad y centralidad.

3 Según Moreno Jiménez (1991), los estimadores focales o densidad de Kernel es una representación cartográfica que permite enriquecer la información en un diagrama de dispersión que adopta enfoques no paramétricos. Esta densidad es utilizada para determinar la densidad de distintos tipos de elementos en un espacio geográfico. los efectos estructurantes de las redes viales en la dinámica del territorio.

En esa perspectiva, el presente trabajo se propone analizar la red vial intermunicipal del microsistema regional de la provincia Centro del departamento de Boyacá para establecer funcionalidades, centralidades y desequilibrios presentes en su configuración espacial y, en segunda medida, identificar patrones de accesibilidad relativa que permitan considerar potencialidades y ventajas comparativas en términos de localización y dinámica vial de los municipios que la integran. El documento se estructura en tres secciones: en la primera se enuncia el contexto del problema y la descripción del área de estudio; en la segunda se expone el enfoque y el contexto metodológico; y en la tercera se presentan los resultados y las conclusiones.

\section{Problema}

La red vial constituye un elemento esencial para entender la configuración espacial de un territorio. Su cobertura se traduce no solo en mayores facilidades para el intercambio económico y regional, sino que en esta converge el equilibrio o las desigualdades que pueden darse en los sistemas territoriales (López-Escolano, Pueyo, Postigo y Alonso, 2016). Una adecuada y suficiente dotación de infraestructuras viales contribuye a mejorar las condiciones y las deficiencias de acceso y de conectividad entre los principales centros urbanos. Estos elementos se consideran básicos y complementarios, toda vez que cumplen con un doble propósito, integrar territorialmente y articular otros modos del sistema de transporte, por lo que son esenciales en el desarrollo socioeconómico y territorial (Herce, 2013).

La red vial generalmente condensa modelos territoriales precedidos por las mismas lógicas económicas, 
como resultado de la creciente interacción de los centros urbanos y la especialización de sus servicios, que dictan la forma como se configura la dinámica territorial en términos de su funcionalidad (Martínez, Mohíno, Ureña y Solís, 2014). Su dotación influye "indirectamente en el funcionamiento socioeconómico de los espacios territoriales al aumentar o reducir las barreras comerciales variando la situación de muchos lugares en función de su localización geográfica” (Loyola y Rivas, 2014, p. 256).

La relación entre las redes de infraestructura de transporte y la accesibilidad ha sido el centro de los diferentes discursos sobre la planificación del transporte, el crecimiento, la cohesión y la sostenibilidad (Fárinos, 2007; Batty, 2009; Rodríguez y Gutiérrez Puebla, 2012). Es un indicador que permite explorar y describir estructuras metropolitanas, regionales y sistemas urbanos para entender aspectos territoriales, como la estructura económica, la concentración y los patrones de especialización (Martínez et al., 2014). El departamento de Boyacá, conformado por 123 municipios y 13 provincias, se caracteriza por ostentar una de las redes viales más amplias del país: se estima que tiene 9.349,07 $\mathrm{km}$ aproximadamente, distribuidos en las redes primaria, secundaria y terciaria, de acuerdo con cifras del Ministerio de Transporte del año 2011.

Su vocación agropecuaria, minera y turística evoca amplios escenarios de desarrollo territorial que deben compatibilizarse con el desarrollo de una infraestructura de transporte vial adecuada y eficiente, en virtud de mejores estadios de conectividad y accesibilidad para el territorio en general. No obstante, según el Plan de Desarrollo de Boyacá 2016-20194 (Gober-

4 El componente de transporte y logística del departamento de Boyacá se fundamenta en el plan de infraestructura para la prosperidad de los Planes Nacionales de Desarrollo 2010-2014, reconocido como el paquete más ambicioso nación de Boyacá, 2016), los modos de transporte (carretero, ferroviario, aeroportuario) y la logística no se encuentran articulados entre sí, lo que ha generado grandes limitaciones para el fortalecimiento de la intermodalidad y la complementariedad de la infraestructura. El microsistema regional de la provincia Centro no se escapa a dicha realidad, los corredores viales intermunicipales no se articulan a un sistema homogéneo y complementario de infraestructuras viales y esto se relaciona con problemas de movilidad, accesibilidad, conectividad, mayor tiempo de viaje, costos de transporte, vulnerabilidad en épocas de invierno e insuficiencia de servicios de transporte público de pasajeros.

La importancia de todos y cada uno de los aspectos señalados es de interés para el transporte de los usuarios, los planificadores territoriales y el sistema en general, puesto que son factores que reflejan la presencia de desequilibrios en las diferentes escalas de uso y funcionalidad del territorio (Freiria et al., 2015). En ese sentido, el presente trabajo se centra en dos aspectos principales: la accesibilidad y la conectividad como atributos espaciales inherentes a la estructura de la red vial de transporte terrestre que permiten evaluar el grado de articulación del sistema, y su funcionalidad en términos de los municipios que lo integran.

\subsection{Descripción del área de estudio}

El microsistema regional de la provincia Centro en Boyacá, Colombia, está conformado por 15 municipios localizados en el departamento de

en materia de infraestructura vial del país, que busca el desarrollo del multimodalismo en proyectos carreteros, férreos, fluviales y puertos. Así mismo, busca desarrollar una infraestructura competitiva que consolide la red vial nacional, fortalezca su conectividad y accesibilidad territorial, acorde con las necesidades y realidades de las regiones. 
Boyacá. El orden poblacional se estima entre los 3.000 y los 190.000 habitantes para el año 2016, según proyecciones del Departamento Administrativo Nacional de Estadística (DANE, 2012). Esta provincia ocupa el $4 .^{\circ}$ puesto en el marco de clasificación de las áreas más dinámicas del sector económico y productivo de las 13 provincias existentes en el departamento (Forero, 2012). La red vial posee una longitud aproximada de 4.440,87 km distribuidos en red secundaria y terciaria, y tiene una extensión territorial de 1.716,3 $\mathrm{km}^{2}$. La provincia es entendida como "unidad histórico-funcional conformada por municipios de características homogéneas con fundamento histórico" (Estupiñan, 2014, p. 167). Se localiza en la región centro del departamento y se caracteriza por sus actividades agrícolas, sociales, de servicios e industriales (Arias, 2010).

\section{FIGURA 1.}

Localización del área de estudio.

Fuente: elaboración propia a partir de cartografía digital de los municipios.

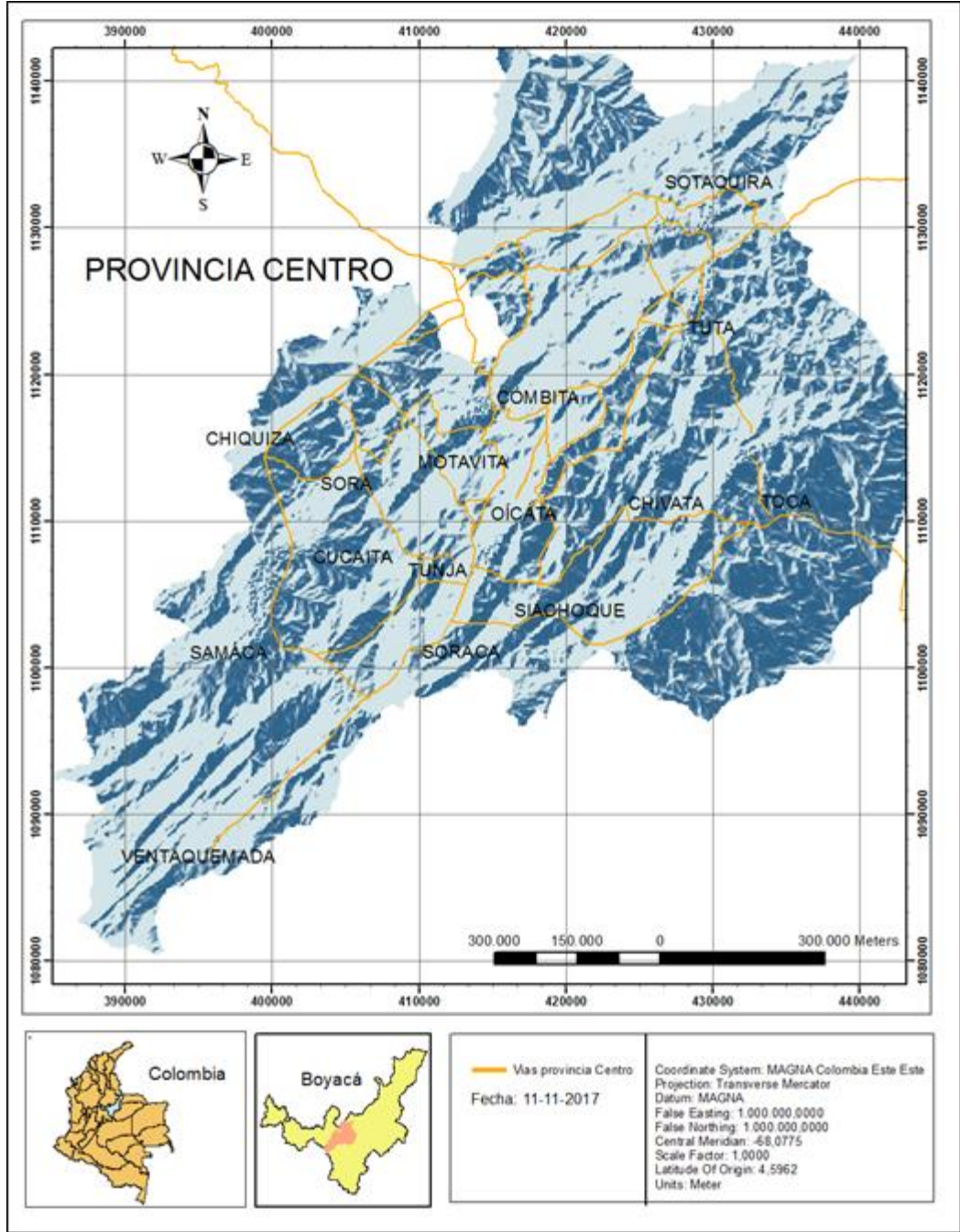




\section{Enfoque teórico-metodológico}

La representación topológica de una red de transporte es similar a una red de nodos y enlaces en la cual las calles, las carreteras, las vías férreas y las combinaciones intermodales son mayoritariamente modeladas como estructuras que definen una topología de red. "Tales topologías pueden tener una forma regular e irregular" (Zhang, Miller-Hooks y Denny, 2015, p. 37). La topología proporciona una posición relativa y, de forma general, cuantitativa de superficies que varían de acuerdo a las relaciones espaciales que se desarrollan entre diferentes lugares (nodos) que por su dinámica mantienen vínculos a través de los cuales tienen acceso (grafo-enlaces) (Brelsford, Martin, Hand y Bettencourt, 2015).

El análisis topológico adopta diferentes enfoques disciplinares: desde la geografía, se vinculan los trabajos de Garrison y Marble (1964), Hagget y Chorley (1969), Potrykowski y Taylor (1984), Seguí y Petrus (1991), Bosque (1997), Cardozo et al. (2009) y Loyola y Rivas (2014), cuyos estudios permitieron la introducción de la teoría de grafos ${ }^{5}$ al análisis de redes de transporte. Su aplicación, todavía de innegable interés, cobra especial relevancia dado que representa un instrumento ideal para las tareas de decisión, teniendo en cuenta su capacidad de abstracción y simplificación. Entre los antecedentes más recientes, se relacionan trabajos sobre el crecimiento y la evolución de la red de carreteras (grafo de vecindad relativa), el modelo topológico de red de calles urbanas, la resiliencia

5 La teoría de grafos, desarrollada por Leonhard Euler en 1736, define al conjunto no vacío de objetos, llamados vértices (o nodos), y una selección de pares de vértices, llamados aristas (edges en inglés), que pueden ser orientados o no. Típicamente, un grafo se representa mediante una serie de puntos (los vértices) conectados por líneas (aristas). de la red vial en términos de su conectividad y la dinámica de la red a través de patrones topológicos (Jiang, 2007; Jingyi y Yifang, 2013; Freiria et al., 2015; Zhao, Sun, Wu, Gao y Liu, 2016).

Las redes de transporte, por su carácter espacial y temporal, configuran uno de los sistemas con mayor incidencia en la dinámica territorial, en tanto que la funcionalidad vial (capacidad y servicio), la conectividad (vínculos y mercados) y la accesibilidad (tiempos de viaje) son elementos que definen la distribución de flujos y la interacción en el territorio, por lo tanto, pueden ser representados mediante nodos y enlaces como una abstracción de la estructura espacial del sistema urbano regional (Patiño y Salazar, 2016). En este contexto, la asociación de una red de transporte como nodos y grafos conectados permite identificar patrones espaciales o estructuras particulares, que suelen abordarse principalmente bajo un análisis descriptivo-explicativo. La red queda compuesta entonces "por elementos lineales, permanentes o temporales y nodales necesarios para la organización de flujos y para el funcionamiento en que está inserta" (Loyola y Rivas, 2014, p. 256).

Como sostiene Somarribas (2008), el análisis topológico es aplicable al conocimiento de la dinámica de las redes de transporte no solo desde la incidencia de este en la forma y las conexiones del territorio, sino porque su estructura emana condiciones particulares, desigualdades y desequilibrios espaciales que es posible estudiar a través de la teoría de grafos y los sistemas de información geográfica (Cardozo, et al., 2009). La aplicación de la teoría de grafos facilita no solo la identificación de problemáticas espaciales entre redes de transporte y centros principales a partir de sus propiedades topológicas, sino que particulariza categorías como la distancia, la distribución y la jerarquía, elementos necesarios 
para comprender las interacciones que establece la red con su espacio topológico.

Desde esta perspectiva, los índices topológicos de accesibilidad y conectividad resultan apropiados, dada su utilidad, para evaluar e identificar patrones y características particulares de la red vial de transportes (Grubesic, Matisziw, Murray y Snediker, 2008). Estas medidas aportan al análisis espacial de la red, de tal forma que cuantifican la capacidad que esta ofrece en términos de su servicio y su configuración espacial. Así mismo, permite generar explicaciones de las realidades territoriales en relación con la integración del sistema vial de transporte terrestre y su articulación con la estructura espacial; de allí que su construcción topológica favorezca no solo lecturas de la insuficiencia de la red, sino que orienta nuevas directrices para potenciar sus relaciones.

\subsection{Medidas de conexión}

Las medidas de conexión permiten establecer la relación y los vínculos entre dos nodos específicos. Sus relaciones indican el alcance de un nodo respecto al otro e indican el número de conexiones posibles. De acuerdo con Madrid y Ortiz (2005) y Grubesic et al. (2008), entre los índices topológicos más utilizados se pueden nombrar los siguientes:

Índice beta $(\beta)$ : describe el grado de conexión de la red a través del número de arcos sobre el número de nodos. Cuando su valor es equivalente a 0 , indica una red nula. Si su valor es igual a 1, la red es de un solo circuito, y de 1 a 3 significa que son redes complejas; puede decirse que a mayor número de arcos, mayor es la conexión que representa.

$$
\mathrm{I} \beta=\mathrm{N}^{\circ} \operatorname{Arcos} / \mathrm{N}^{\circ} \text { Nodos }
$$

Índice gamma $(\gamma)$ : se obtiene a partir de dividir el número de arcos existentes en la red entre el máximo posible de arcos sobre el número real de nodos de la red. Su cercanía a un valor de 1 representa una red idealmente más conectada.

$$
\mathrm{I} \gamma=2 / \mathrm{N}(\mathrm{n}-1)
$$

Índice gamma (\%): señala el valor porcentual de arcos que debe introducirse en cada nodo para obtener una red más integrada. Un valor cercano al $100 \%$ supone una red ideal, lo cual está alejado de la realidad.

$$
\mathrm{I} \gamma=(\mathrm{a} / 3(\mathrm{n}-2)) * 100
$$

Número ciclomático $(\mu)$ : corresponde al número de circuitos presentes en un grafo. Un circuito es cada una de las maneras de ir desde un nodo hasta el mismo sin tener que pasar dos veces por la misma arista.

$$
\mathrm{NC}=(\mathrm{a}-(\mathrm{n}-1))
$$

Índice alfa $(\alpha)$ : se obtiene de la relación entre el número de circuitos existentes y el máximo posible de la red.

$$
\alpha=\mathrm{NC} / 2(\mathrm{~N}-5)
$$

\subsection{Medidas de accesibilidad}

En el análisis topológico, la accesibilidad es un índice que define el número de veces que es necesario atravesar un arco para llegar a un nodo de referencia. En consecuencia, los nodos tendrán mayor jerarquía cuando esta cantidad es inferior o posibilita mayor facilidad para acceder de uno a los restantes. Para establecer la accesibilidad de una red, se construye una matriz de accesibilidad topológica en una tabla binaria de doble entrada con la asignación de grafos y nodos correspondientes y se hace una relación por el camino más corto, lo cual indica que cada arco adquiere un valor de 1; 
adicionalmente, se disponen dos casillas más con el número asociado y el número de Shimbel.

De acuerdo con Cardozo et al. (2009), para la construcción de esta matriz es necesario elaborar una matriz de conectividad, que se desarrolla de manera similar a la anterior, cuyo valor permite sintetizar únicamente los nodos que tienen relación directa.

Número asociado (NS): se refiere a la distancia topológica expresada en el número de arcos que es necesario recorrer para alcanzar el nodo más distante por el camino más corto. Señala que cuanto más bajo es el número, más alto es el grado de accesibilidad. Este número se identifica en la matriz de accesibilidad por ser el mayor en cada fila.

Número de Shimbel (SHI): se obtiene sumando los valores de cada fila en la matriz de accesibilidad. Representa el número de arcos que es necesario atravesar desde un nodo a los demás por el tramo más corto. Es más accesible el nodo que presente el índice más bajo, y tiene una expresión de:

$$
\text { Shimbel }=\sum \mathrm{d} x y
$$

Índice de omega o dispersión: se haya para cada nodo de la accesibilidad topológica relativa. Se obtiene sumando el número de arcos que separa cada nodo de todos los demás por el tramo más corto.

$$
\Omega=\mathrm{SHI}^{*} \mathrm{SHI}_{\mathrm{i}} / \mathrm{SHI}^{\mathrm{i}}-\mathrm{SHI}_{\mathrm{i}}
$$

Una vez establecidos estos valores en la matriz de accesibilidad topológica, es posible determinar las distancias ideales $(d i)$ y las distancias reales $(d r)$, las cuales constituyen otra alternativa para conocer la eficiencia de la red.

Accesibilidad ideal: se calcula a partir de la sumatoria de la distancia lineal entre localidades:

$$
\mathrm{AI}_{\mathrm{i}}=\Sigma \mathrm{d}_{\mathrm{i}} \mathrm{l}
$$

Donde $\mathrm{AI}_{\mathrm{i}}$ : accesibilidad ideal para cada asentamiento, y díil: es la distancia de la localidad $i$ con respecto a localidades $j$.

Accesibilidad real: se calcula a partir de la sumatoria de las distancias reales a través de las vías de comunicación entre localidades. Su expresión es:

$$
\mathrm{AR}_{\mathrm{i}}=\Sigma \mathrm{dr}_{\mathrm{i}} \mathrm{l}
$$

Donde $\mathrm{AR}_{\mathrm{i}}$ : accesibilidad real para cada asentamiento; $\mathrm{dr}_{\mathrm{i}} \mathrm{\imath}$ : la distancia de la localidad $i$ con respecto a localidades $j$. Siguiendo a Principi (2012), estos índices generan un conocimiento global de las distancias, entendidas como la forma de ponderar aquellos nodos o localidades que mejores condiciones de accesibilidad presentan.

Razón de sinuosidad: es una medida que permite clasificar el tipo de red en relación con su forma real. Se calcula de la siguiente manera:

$$
\mathrm{S}=\mathrm{Lr} / \mathrm{Lg}
$$

Donde Lr: longitud real de la red/Lg, longitud del grafo.

La combinación del análisis topológico y los sistemas de información geográfica (SIG) no comporta una idea nueva ya que este tipo de ejercicios viene incorporándose con mucho acento. Principalmente se enfocan al análisis de la vulnerabilidad de la red vial mediante la técnica biclustering, que identifica patrones espaciales de la red a través del índice de alfa (Freiria et al., 2015). Respecto a los estimadores focales Kernel, se pueden citar los trabajos de De Cos Guerra (2004), Cardozo et al. (2009), Yu, Ai y Shao (2015) y Arias, Cardozo y Parras (2016). 
Estimadores Kernel o densidades focales: es una representación cartográfica que enriquece la información en un diagrama de dispersión que adopta enfoques no paramétricos (Moreno Jiménez, 1991). Se expresa mediante la función:

$$
\hat{\mathrm{f}}(\mathrm{x})=\frac{1}{\mathrm{nh}} \sum_{\mathrm{i}=1}^{\mathrm{n}} \mathrm{K}\left(\frac{\mathrm{x}-\mathrm{x}_{\mathrm{i}}}{\mathrm{h}}\right)
$$

El valor de la función de intensidad Kernel en un punto o píxel se obtiene mediante un radio o alcance determinado. Para este ejercicio, se basó en los valores porcentuales del índice de omega o accesibilidad topológica (expuesto en la matriz de accesibilidad topológica), donde se hace una triangulación en un radio de 10 kilómetros del cual se obtiene una imagen por el porcentaje de densidad de accesibilidad relativa.

\subsection{Materiales y métodos}

El estudio se fundamenta en la red vial intermunicipal secundaria y terciaria, según categorías del Ministerio de Transporte (2011). Para esto, se sobrepone el conjunto de líneas y puntos de la red vial intermunicipal de las cabeceras municipales de los 15 municipios que conforman la unidad de análisis. Se obtuvieron 52 nodos y 97 arcos, de los cuales 26 aproximadamente son relaciones directas. La construcción de las matrices topológicas consta de 52 filas y 52 columnas para cada uno de los nodos identificados; estos representan los 15 municipios y los 37 cruces o intersecciones que conforman la red vial real. Una vez se simplificada la red vial en su representación topológica, se obtiene el NS, el SHI, el índice omega y el índice de dispersión para las salidas cartográficas respectivas.

\section{Resultados}

En la Tabla 1 se presentan las cifras de los índices de conexión topológica. La conexión máxima de la red vial intermunicipal en la provincia Centro es de $1,8(\beta)$, esto significa una red compleja, toda vez que los valores de beta $(\beta)$ y gamma $(\gamma)$ deben estar próximos a 1. Esto quiere decir que la red vial no está idealmente bien conectada y debe introducir un $64 \%$ de aristas para lograr un máximo de conectividad y una red más integrada.

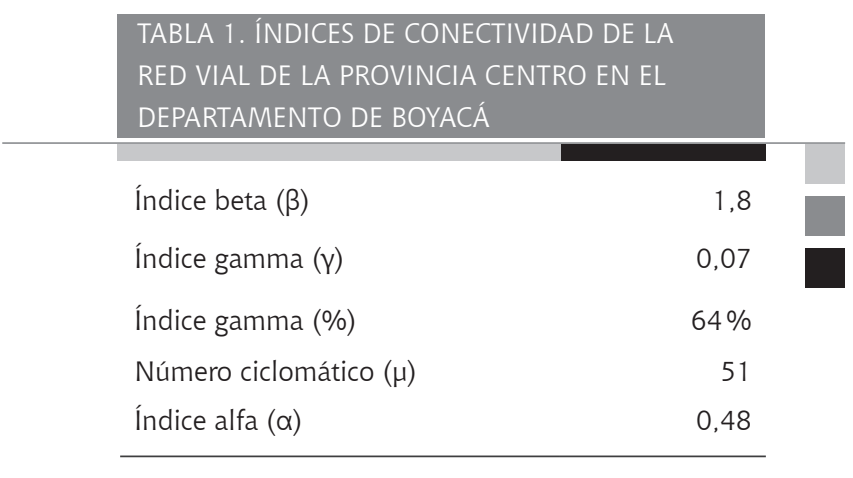

Fuente: elaboración a partir de cálculos de red topológica.

De acuerdo con el índice o número ciclomático $(\mu)$, el número de circuitos equivale a 51, que, comparado con el valor ideal 94, entrevé la complejidad de la red respecto al número de aristas o grafos que sería necesario recorrer sin tener que remitirse nuevamente a los mismos. Esto significa que el índice de alfa $(\alpha)$, cuyo valor máximo es de 1 , diste entre los circuitos presentes y el número máximo posible de ellos, puesto que su valor es de 0,48 , lo cual indica que el desarrollo de la conectividad de la red vial de la provincia Centro es insuficiente frente a los parámetros establecidos cuando se trata de un grafo completamente conectado. 


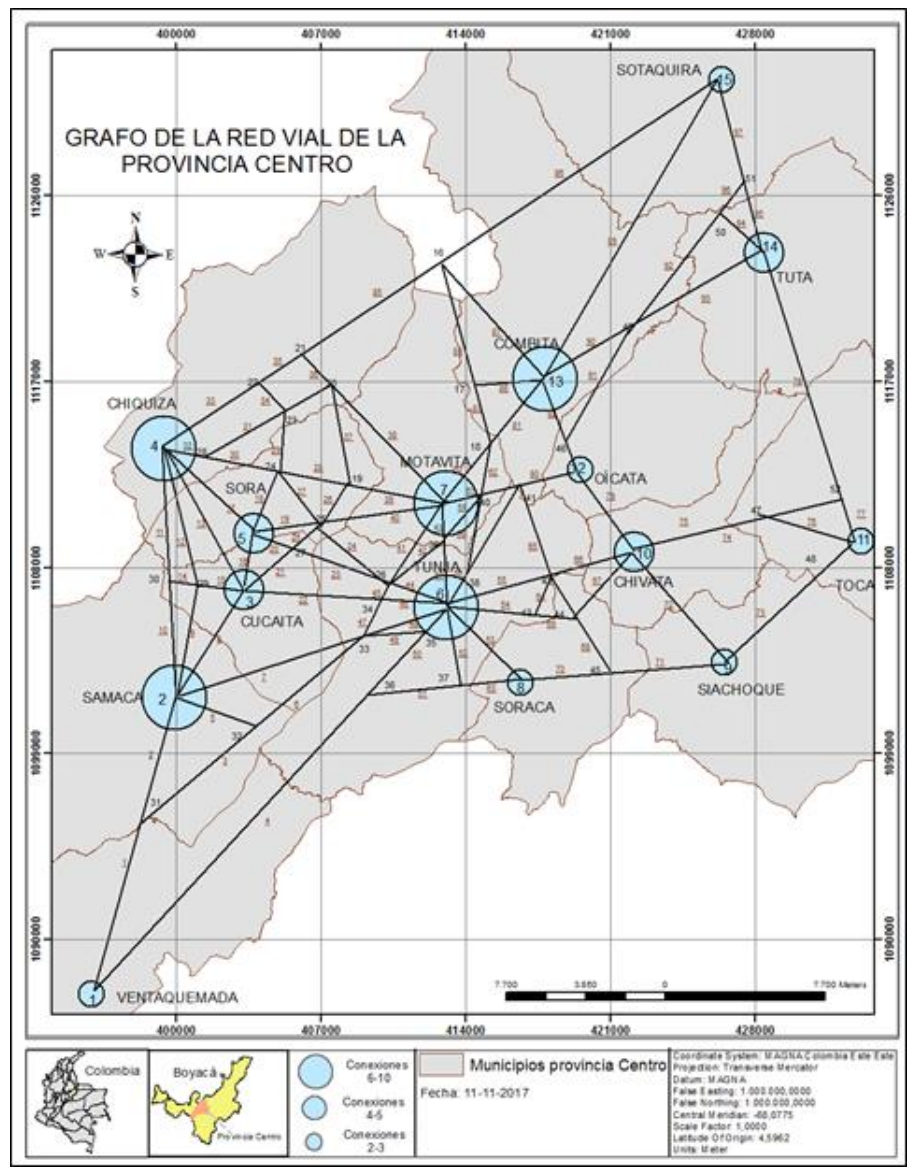

\section{FIGURA 2.}

Número asociado (NS) de la red vial del microsistema regional de la provincia Centro.

Fuente: elaboración propia a partir de matriz de accesibilidad topológica de la red vial de la provincia Centro.

Teniendo en cuenta lo anterior, la Figura 2 pone en evidencia patrones de conexión claramente diferenciables entre cada uno de los 15 municipios. La red topológica de la provincia Centro muestra una tendencia a la concentración y la dispersión del sistema en donde prevalece la centralidad de unos nodos respecto a otros según sus conexiones, lo que demuestra el bajo grado de integración de la red en términos de las relaciones directas entre cabeceras municipales.

\section{TABLA 2. NÚMERO DE RELACIONES DIRECTAS DE CADA UNA DE LAS CABECERAS} MUNICIPALES EN LA PROVINCIA CENTRO EN EL DEPARTAMENTO DE BOYACÁ

$\begin{array}{lclr}\text { Cabeceras municipales } & \mathbf{N}^{\circ}{ }^{\circ} \text { de conexiones } & \text { Cabeceras municipales } & \mathbf{N}^{\circ} \text { de con } \\ \text { Ventaquemada } & 2 & \text { Siachoque } & 3 \\ \text { Samacá } & 6 & \text { Chivatá } & 5 \\ \text { Cucaita } & 5 & \text { Toca } & 3 \\ \text { Chíquiza } & 6 & \text { Oicatá } & 3 \\ \text { Sora } & 5 & \text { Cómbita } & 6 \\ \text { Tunja } & 10 & \text { Tuta } & 4 \\ \text { Motavita } & 7 & \text { Sotaquirá } & 3 \\ \text { Soracá } & 3 & & \end{array}$

Fuente: Elaboración a partir de matriz de conectividad topológica. 
De la matriz de conectividad topológica, se puede señalar que entre los nodos mejor conectados se encuentra la ciudad de Tunja y los municipios de Motavita, Samacá, Chíquiza y Cómbita, caracterizados por ostentar entre seis y diez enlaces directos. Cabe destacar que Tunja es la entidad territorial mejor conectada, con potencialidades importantes por disponer de mejores condiciones de integración en el marco de la estructuración topológica de la red.

En un segundo término se encuentran las cabeceras que evidencian cuatro y cinco enlaces $\mathrm{y}$, de otro lado, aquellas que presentan dos y tres enlaces, cuyo número de conexiones tendría fuertes implicaciones en la dinámica y la configuración de la red vial, relacionadas con la distancia y la dispersión. Este escenario permite considerar que solo cinco de los 15 municipios de la provincia Centro cuentan con conexiones directas que favorecen su conectividad al sistema vial regional, y, en ese caso, su articulación a la funcionalidad del territorio en general. En consecuencia, la red vial no responde a una configuración uniforme, ya que su conectividad topológica, medida por el número de conexiones, evoca claros desequilibrios entre cada uno de los municipios analizados.

\section{TABLA 3. CABECERAS MUNICIPALES SEGÚN NÚMERO ASOCIADO (NS), NÚMERO SHIMBEL} (SHI) E ÍNDICE DE OMEGA

\begin{tabular}{lccc}
\multicolumn{1}{c}{ Cabeceras municipales } & NS & SHI & Índice omega \\
Ventaquemada & 10 & 215 & $37 \%$ \\
Samacá & 8 & 203 & $30 \%$ \\
Cucaita & 9 & 244 & $54 \%$ \\
Chíquiza & 9 & 235 & $48 \%$ \\
Sora & 9 & 236 & $49 \%$ \\
Tunja & 7 & 155 & $2 \%$ \\
Motavita & 6 & 157 & $3 \%$ \\
Soracá & 8 & 198 & $27 \%$ \\
Siachoque & 7 & 165 & $8 \%$ \\
Chivatá & 9 & 190 & $23 \%$ \\
Toca & 10 & 251 & $58 \%$ \\
Oicatá & 9 & 195 & $25 \%$ \\
Cómbita & 7 & 196 & $26 \%$ \\
Tuta & 9 & 152 & $20 \%$ \\
Sotaquirá & 8 & 229 & $45 \%$ \\
Índice de dispersión & & 11.261 & \\
Longitud media del grafo & & 213 &
\end{tabular}

Fuente: elaboración propia a partir de la matriz de accesibilidad topológica. 
Respecto de la accesibilidad, según el número asociado (NS), las cabeceras más accesibles en términos topológicos se encuentran en la ciudad de Tunja y el municipio de Cómbita, cuyos valores son inferiores en relación al número de arcos que es necesario recorrer para alcanzar el nodo más distante por el camino más corto. El número de Shimbel marca una tendencia similar respecto a los mismos nodos, puesto que aquellos valores inferiores a la longitud media del grafo se consideran como accesibles; en este caso, y de acuerdo con la Tabla 3 , aproximadamente el $60 \%$ de cabeceras cumple con esta característica. No obstante, el índice de omega $(\Omega)$ denota porcentualmente la distribución en términos de la accesibilidad que presenta cada cabecera analizada, de donde se puede observar la densidad de accesibilidad relativa según los estimadores de Kernel (ver Figura 3).
FIGURA 3.

Densidad de accesibilidad relativa en el microsistema regional de la provincia Centro.

Fuente: elaboración propia a partir de la matriz de accesibilidad de la red vial de la provincia Centro.
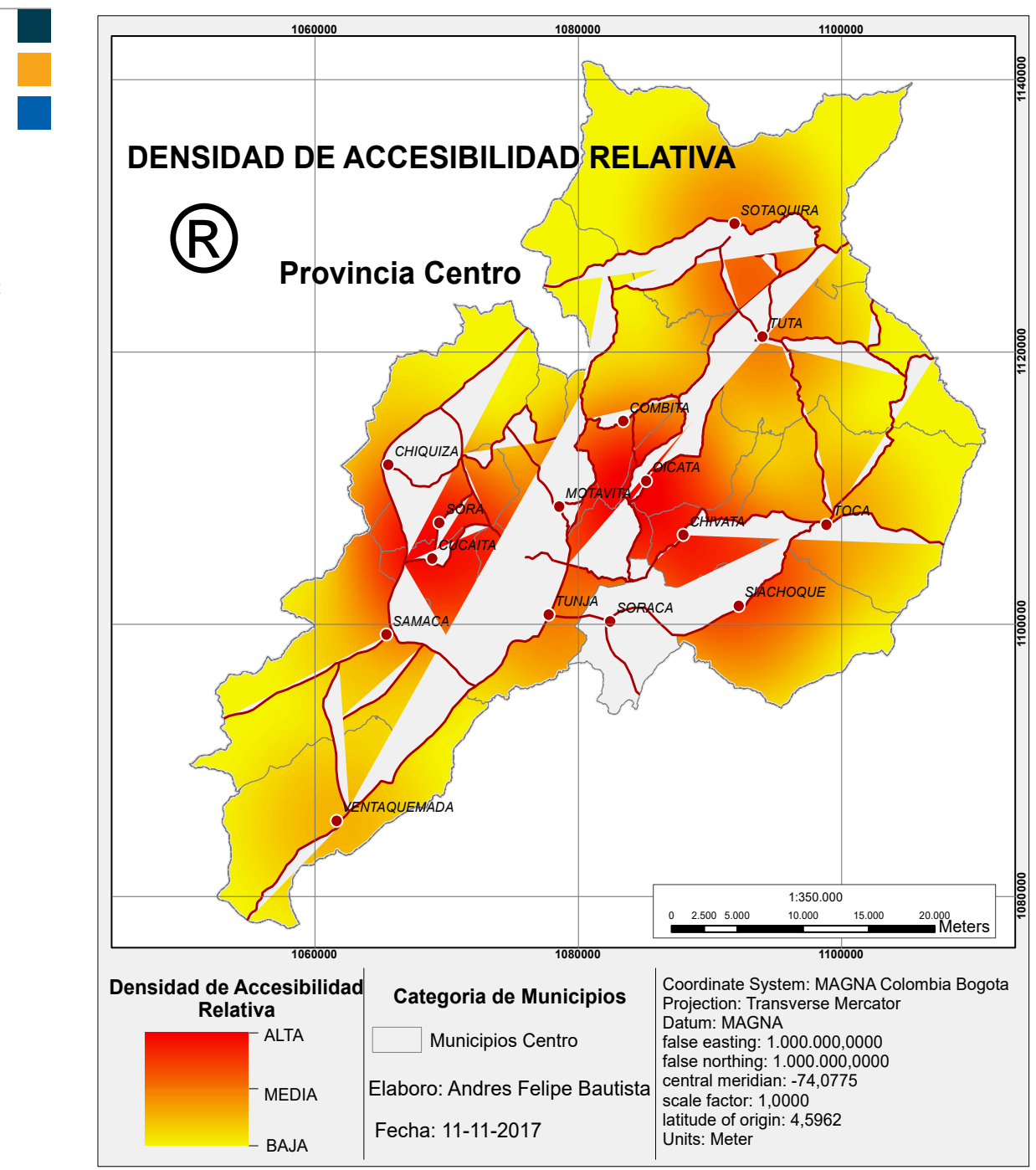
De acuerdo con lo anterior, la red vial posee un carácter regular, dado que el $60 \%$ de las cabeceras o nodos son accesibles y el otro $40 \%$ no cuenta con un escenario deseado de accesibilidad. Si se relaciona este aspecto con el índice de densidad media $444,87 \mathrm{~km} / 1.716,3 \mathrm{~km}^{2}$ implica una densidad vial de $0,259 \mathrm{~km} / \mathrm{km}^{2}$ y una razón de sinuosidad equivalente 1,2 , lo cual refleja una red regular con un valor de sinuosidad representativa, que permite considerar la existencia de tramos viales con bastante curvatura que limitan la accesibilidad y la conectividad ideal.

En este contexto, se puede decir que las cabeceras localizadas en la parte central de la provincia conjugan mayores posibilidades de accesibilidad relativa, teniendo en cuenta que es allí donde se fija la intensidad de los menores valores de accesibilidad, de acuerdo con la Figura 3. Estas cabeceras definen los patrones de densidad más fuertes como consecuencia de su contigüidad espacial. Un ejemplo de ello es el caso de Tunja, Motavita, Cómbita y Oicatá, cuyo índice de accesibilidad real $\left(\mathrm{AR}_{\mathrm{i}}\right)$ no supera los 16 $\mathrm{km}$ de un nodo respecto al otro. Cabe señalar que estos municipios se inscriben en un sistema vial lineal porque se vinculan directamente a uno de los corredores viales de mayor importancia estratégica para la región y el departamento: se trata de la doble calzada Bogotá-Tunja-Sogamoso, que ostenta una demanda de tráfico promedio diario semanal (TPDS) de 2.500 vehículos en esta dirección, según el Instituto Nacional de Vías (Invias) (Ministerio de Transporte, 2011).

Frente a las demás cabeceras municipales, la densidad de accesibilidad relativa varía en su intensidad, particularmente para aquellos municipios localizados en los externos de la provincia, cuyo valor de accesibilidad está por encima de la longitud media del grafo (213), como en el caso de Ventaquemada,
Sotaquirá, Cucaita, Chíquiza, Sora y Toca, lo que hace que el sistema denote polarizaciones y dispersiones asociadas a la distancia y la cobertura de la red vial. De esta forma, se reducen no solo las posibilidades de integración topológica en términos de accesibilidad ideal, sino que se refuerzan las centralidades de aquellas cabeceras municipales que presentaron mejores características de conexión.

Lo anterior demuestra que la red vial de la provincia Centro no es uniforme en términos de la conectividad y la accesibilidad ofrecidas hacia todos los nodos o cabeceras municipales, lo que permite considerar la presencia de desequilibrios en la misma prestación del servicio y la distribución espacial de la infraestructura. Su configuración responde a particularidades propias de la centralidad de algunos municipios que por su localización se ven mayormente favorecidos en la conectividad vial regional y que pueden inducir patrones espaciales localizados en el microsistema regional, que se traducen en impactos socioeconómicos y en algunas problemáticas relacionas con la movilidad y con la complementariedad que supone la red.

Finalmente, la importancia de conocer la accesibilidad y la conectividad a través del análisis topológico es que estas medidas, más allá de su expresión espacial en términos absolutos y relativos, ofrecen una aproximación simplificada de la estructura vial topológica de la provincia, cuya accesibilidad y conectividad están supeditadas a la dotación y la distribución de infraestructuras viales y los diferentes modos de transporte utilizados (Escobar y Urazán, 2014). No obstante, el resultado es una red vial distribuida de forma irregular, que sectoriza la funcionalidad del sistema y condiciona los servicios y el desarrollo de relaciones entre municipios. 


\section{Conclusiones}

El análisis de redes aplicado a la red vial de la provincia Centro en el departamento de Boyacá es el resultado de una primera aproximación a una realidad territorial poco estudiada. Las medidas de accesibilidad y conectividad expuestas, aun cuando no son los únicos indicadores de medición, son instrumentos que permiten comprender la estructura territorial de la provincia en términos de sus relaciones y vínculos intermunicipales. Del análisis se obtienen resultados importantes, que manifiestan claras centralidades prácticamente para la mitad de los municipios que conforman el microsistema regional, lo que pone en evidencia la presencia de desequilibrios regionales expresados en el aislamiento, la polarización y la especialización de algunos municipios en la prestación de servicios, lo cual tiene fuertes implicaciones para los núcleos poblacionales.

La incorporación del índice de densidad de Kernel es otro de los instrumentos de este ejercicio que permite considerar la situación real de los niveles de accesibilidad relativa y del cual se obtuvo una imagen que afirma la irregularidad de la red vial, que no ofrece una adecuada articulación y cuyas condiciones de estado, capacidad y, particularmente, niveles de servicio no son uniformes, puesto que el $60 \%$ de los municipios son accesibles y el otro $40 \%$ no cuenta con un escenario deseado de accesibilidad.

En cuanto a los niveles de conectividad, la red vial presenta mínimos potenciales para más de la mitad de las cabeceras municipales al considerar que los índices de alfa $(\alpha)$ y beta $(\beta)$ son distantes al promedio normal de un grafo idealmente conectado. Adicionalmente, el índice de sinuosidad refleja la saturación de relaciones directas, sumado al agrupamiento según número asociado (NS) de valores altos en el número de enlaces de la mayoría de municipios. Esto supone, por un lado, mejores oportunidades de relación y complementariedad de la red vial para municipios como Tunja, Motavita, Oicatá y Cómbita, que disponen de mejores condiciones de integración en el marco de la estructuración topológica de la red, y, por otro lado, presenta fuertes implicaciones territoriales, relacionadas con la distancia y la dispersión, para las demás cabeceras.

Analizar la red vial de transporte terrestre mediante la combinación de la teoría de grafos y los sistemas de información geográfica constituye no solo una de las ventanas metodológicas de mayor interés en los estudios del transporte porque estima los escenarios de accesibilidad, sino que es una herramienta que permite visualizar los efectos estructurantes que supone la dotación de infraestructuras viales en el territorio y dar cuenta de los desequilibrios presentes. Además, es un instrumento útil para la planificación, puesto que pone de presente la conjugación de métodos y procesos válidos para generar escenarios de mejora. Es de señalar que el análisis debe complementarse con la inclusión de más variables y criterios (costos, tiempos de desplazamiento, frecuencias de viaje y servicios de transporte) que amplíen el conocimiento de la realidad de la provincia Centro. 


\section{Referencias}

Alvarez-Palau, E. J. y Aguilar, A. (2015). Accesibilidad territorial ferroviaria y distribución de población: Inglaterra y Gales, 1871-1931. GeoFocus, 15, 75-104. Recuperado de http://www.geofocus.org/index.php/geofocus/article/view/414

Arias, C, Cardozo, O. y Parras., M. (2016). Análisis de conectividad y densidad de la red vial en la reserva natural del Iberá con sistemas de información geográfica (SIG). Revista Geográfica Digital, 13(26), 1-16. Recuperado de http://hum.unne. edu.ar/revistas/geoweb/Geo26/contenidos/cardozo26.htm

Arias, H. (2010). Aglomeración económica y sistemas locales de trabajo en Boyacá. Apuntes del Cenes, 30(51), 9-43. Recuperado de http://revistas.uptc.edu.co/index. php/cenes/article/view/32/33

Batty, M. (2009). Accessibility: in search of a unified theory. Environment and Planning B: Planning and Design, 36(2), 191-194. Recuperado de http://journals.sagepub. com/doi/abs/10.1068/b3602ed?id = b3602ed\&

Bautista, A. F. y Cerquera, F. A. (2014). Procesos espaciales asociados a la construcción de la doble calzada BTS en los municipios de Tunja, Cómbita y Oícata (20052012). Perspectiva Geográfica, 19(2), 219-240. Recuperado de http://revistas.uptc. edu.co/revistas/index.php/perspectiva/article/view/4092

Bellet, C. (2013). Transporte y desarrollo territorial. El estudio de los efectos asociados a la implantación de alta velocidad ferroviaria a través del caso español. Revista Transporte y Territorio, 8, 117-137. Recuperado de http://dialnet. unirioja.es/servlet/articulo? codigo $=4340460$

Bosque, J. (1997). Sistemas de información geográfica. (2ª ed.). Madrid: Ediciones Rialp.

Brelsford, C., Martin, T. Hand, J. y Bettencourt, L. (2015). The topology of cities. Recuperado de https://pdfs.semanticscholar.org/ b056/7ec801c05a558ebb7533edeaf1bb1c5f6737.pdf

Cardozo, O., Gómez, E. y Parras, M. (2009). Teoría de grafos y sistemas de información geográfica aplicados al transporte público de pasajeros en Resistencia (Argentina). Revista Transporte y Territorio, 1, 89-111. Recuperado de www.redalyc.org/ pdf/3330/333027079005.pdf

Cats, O. (2017). Topological evolution of a metropolitan rail transport network: The case of Stockholm. Journal of Transport Geography, 62, 172-183. Recuperado de https://www.sciencedirect.com/science/article/pii/S0966692317300807

Chías, L. (2008). Análisis espacial de redes de transporte, empleando Sistemas de Información Geográfica. Revistas análisis geográficos, 39, 93-113.

De Cos Guerra, O. (2004). Valoración del método de densidades focales (Kernel) para la identificación de los patrones espaciales de crecimiento de la población en España. GeoFocus, 4, 136-165. Recuperado de http://geofocus.rediris.es/docPDF/ Articulo7_2004.pdf

Departamento Administrativo Nacional de Estadística (DANE). (2012) Proyecciones de la población departamental y municipal 1985-2020. Recuperado de http://www. dane.gov.co/ 
Dupuy, G. (1998). El urbanismo de las redes, teorías y métodos. Barcelona: Editorial Oikos Tau.

Escobar García, D. A. y Urazán Bonells, C. F. (2014). Accesibilidad territorial: instrumento de planificación urbana y regional. Revista Tecnura, 18, 241-253. Recuperado de https://revistas.udistrital.edu.co/ojs/index.php/Tecnura/article/view/8171

Estupiñan, L. (2014). La provincia en Boyacá: unidad territorial, histórico-funcional de planificación en la gestión del desarrollo regional endógeno, 2004-2011. Apuntes del CENES, 33(58), 163-188. Recuperado de http://repository.urosario.edu.co/ bitstream/handle/10336/3017/33375888-2012.pdf

Fárinos, J. (2007). Planificación de infraestructuras y planificación territorial. Gobernanza y gestión de dinámicas multiescalares. Papers: Regió Metropolitana de Barcelona: Territori, estratègies, planejament, 44, 32-43. Recuperado de https://ddd. uab.cat/pub/prmb/18883621n44/18883621n44p32.pdf

Forero, M. (2012). Análisis del cambio poblacional de los municipios del departamento de Boyacá a partir de la aglomeración urbana y la dinámica económica generada en sus principales ciudades. (Tesis de maestría). Pontificia Universidad Javeriana, Bogotá, Colombia. Recuperado de https://repository.javeriana. edu.co/bitstream/handle/10554/15628/ForeroMedinaMariaHulima2012. pdf? sequence $=1$ \&isAllowed $=y$

Freiria, S., Ribeiro, B. y Tavares, A. (2015). Understanding road network dynamics: Link-based topological patterns. Journal of Transport Geography, 46, 55-66. Recuperado de http://bin.t.u-tokyo.ac.jp/kaken/pdf/150619_shibahara.pdf

Garrison, W. y Marble, D. F. (1964). Factor-analytic study of the connectivity of a transportation network. Papers in Regional Science Association, 12(1), 231-238. doi: https://doi.org/10.1111/j.1435-5597.1964.tb01269.x

Grubesic, T., Matisziw, T., Murray, A. y Snediker, D. (2008). Comparative approaches for assessing network vulnerability. International Regional Science Review, 31(1), 88112. doi: https://doi.org/10.1177/0160017607308679

Gutiérrez, J. y Gómez, G. (1999). The impact of orbital motorways on intrametropolitan accessibility: case of Madrid's M-40. Journal of Transport Geography, 7(1), 1-15.

Gobernación de Boyacá. (2016). Plan de Desarrollo 2016-2019. Creemos en Boyacá, tierra de paz y libertad. Recuperado de http://www.boyaca.gov.co/gobernacion/ politicas-planes-y-programas/9498-pdd-boyaca-2016-2019

Hagget, P. y Chorley, R. (Eds.). (1967). Modelos, paradigmas y la nueva geografía. En Modelos en geografía (pp. 19-41). Londres: Methuen.

Herce, M. (1983). La utilización de indicadores topológicos en el análisis de redes de comunicaciones. Ensayo sobre la red de carreteras de Cataluña. Recuperado de https://ddd.uab.cat/pub/dag/02121573n3/02121573n3p3.pdf

Herce, M. (2013). Carreteras para una movilidad equitativa. Revista de Obras

Públicas, 3540, 21-29. Recuperado de http://ropdigital.ciccp.es/detalle_articulo. php? registro $=19234$ \&numero_revista $=3540$ \&anio $=2013$ \&anio_ini $=$ \&anio_fin $=$ 
Jiang, B. (2007). A topological pattern of urban street networks: universality and peculiarity. Physica A, 384(2), 647-655. doi: 10.1016/j.physa.2007.05.064.

Jingyi, L, y Yifang, B. (2013). Complex topology network of transport systems, Transportation Comments. A Transdisciplinary Transnational Journal, 33(6), 658-685. Recuperado de https://www.tandfonline.com/doi/abs/10.1080/01441647.2013. 848955

López-Escolano, C., Pueyo, Á., Postigo, R. y Alonso, M. (2016). Valoración y representación cartográfica de la accesibilidad viaria en la España peninsular: 1960-2014. GeoFocus, 18, 169-189. doi: http://dx.doi.org/10.21138/GF.486

Lotero, L. y Hurtado, R. (2014). Vulnerabilidad de redes complejas y aplicaciones al transporte urbano: una revisión de la literatura. Revista EIA, 11(21), 67-78. Recuperado de http://www.scielo.org.co/pdf/eia/n21/n21a06.pdf

Loyola, C. y Rivas, J. (2014). Accesibilidad a los centros poblados en el valle del Itata, provincia de Nuble, Chile. Polígonos, Revista de Geografía, 26, 255276. Recuperado de https://www.researchgate.net/publication/279171356_ Accesibilidad_a_los_centros_poblados_en_el_Valle_del_Itata_Provincia_de Nuble Chile

Madrid, A. y Ortiz, L. (2005). Análisis y síntesis en cartografía. Algunos procedimientos. Bogotá: Unibiblios.

Martínez, H., Mohíno, I., Ureña, J. y Solís, E. (2014). Road accessibility and articulation of metropolitan spatial structures: the case of Madrid (Spain). Journal of Transport Geography, 37, 61-73.

Martínez-Sánchez., H. (2012). La accesibilidad regional y el efecto territorial de las infraestructuras de transporte. Aplicación en Castilla-La Mancha. Boletín de la Asociación de Geógrafos Españoles, 59, 79-103. Recuperado de https://dialnet. unirioja.es/descarga/articulo/3938147/1.pdf

Ministerio de Transporte, Instituto Nacional de Vías. (2011). Volúmenes de tránsito, series históricas y composición de tránsito promedio diario. Recuperado de https://www.invias.gov.co/index.php/documentos-tecnicos

Miralles Guasch, C. (2013). Presentación: Dossier metodologías y nuevos retos en el análisis de la movilidad y el transporte. Revista Transporte y Territorio, 8, 1-6. Recuperado de http://www.redalyc.org/pdf/3330/333027381001.pdf

Moreno Jiménez, A. (1991). Modelización cartográfica de densidades mediante estimadores Kernel. Treballs de la Societat Catalana de Geografia, 6(30), 155-170. Recuperado de https://repositorio.uam.es/handle/10486/668528

Patiño, B. y Salazar, C. (2016). Proyectos de infraestructura vial e integración territorial. Bitácora Urbano Territorial, 26(2), 79-86. Recuperado de https://revistas.unal.edu. co/index.php/bitacora/article/view/57431/html

Principi, N. (2012). Análisis de accesibilidad e interacción espacial: cálculos cuantitativos y representación cartográfica a escala regional. Geografía y Sistemas de Información Geográfica, 4(4), 23-38. Recuperado de https://www.researchgate. net/publication/313475488_Analisis_de_accesibilidad_e_interaccion_espacial_ calculos_cuantitativos_y_representacion_cartografica_a_escala_regional 
Potrykowski, M. y Taylor, Z. (1984). Geografía del transporte. España: Oikos.

Rodrigue, J. P., Comtois, C. y Slack, B. (2009). The Geography of Transport Systems. Londres: Routledge.

Rodríguez, E. y Gutiérrez Puebla, J. (2012). Análisis de vulnerabilidad de redes de carreteras mediante indicadores de accesibilidad y SIG: intensidad y polarización de los efectos del cierre de tramos en la red de carreteras de Mallorca. GeoFocus, 12, 374-394. Recuperado de http://geofocus.rediris.es/2012/Articulo15_2012.pdf

Seguí, J. y Petrus, J. (1991). La organización espacial de los sistemas de transportes. En Geografía de redes y sistemas de trasportes (pp. 60-74). Serie Espacios y Sociedades, No 16. Madrid: Síntesis.

Serrano, J. M. (2004). Articulación territorial de la Península Ibérica mediante la red de transportes terrestres. Revista de Estudios Regionales, 69, 19-55. Recuperado de http://www.redalyc.org/pdf/755/75506901.pdf

Somarribas, L. (2008). Hacia una geografía de redes: un nuevo paradigma de análisis espacial alternativo al enfoque regional. Revista Geográfica de América Central, 1(41), 1-34. Recuperado de http://www.revistas.una.ac.cr/index.php/geografica/ article/view/1702

Yu, W., Ai, T. y Shao, S. (2015). The analysis and delimitation of Central Business District using network Kernel density estimation. Journal of Transport Geography, 45, 32-47. doi: 10.1016/j.jtrangeo.2015.04.008.

Xie, F. y Levinson, D. (2007). Modeling the Growth of Transportation Networks: A Comprehensive Review. Networks and Spatial Economics, 9(3), 291-307. Recuperado de https://link.springer.com/article/10.1007\%2Fs1 1067-007-9037-4

Xie, F. y Levinson, D. (2009). Topological evolution of surface transportation networks. Computers, Environment and Urban Systems, 33(3), 211-223. Recuperado de https://www.academia.edu/13909158/Topological_evolution_of_surface_ transportation_networks

Zhang, X., Miller-Hooks, E. y Denny, K. (2015). Assessing the role of network topology in transportation network resilience. Journal of Transport Geography, 46, 35-45. Recuperado de https://www.sciencedirect.com/science/article/pii/ S0966692315000794

Zhao, F., Sun, H., Wu, J., Gao, Z. y Liu, R. (2016). Analysis of Road Network Pattern Considering Population Distribution and Central Business District. PloS ONE, 11(3), e0151676. doi: https://doi.org/10.1371/journal.pone.0151676

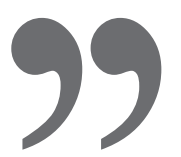

